\title{
Detection of partial occlusions of assembled components to simplify the disassembly tasks
}

\begin{abstract}
An automatic disassembly cell requires of a computer vision system for recognition and localization of the products and each of theirs components. The detection of occlusions adds more information to the knowledge base to identify components and products, and generate a trustworthy and precise relational model (generic graph of hierarchic relations among the different components that make up the product). In this paper, a method to detect partial occlusions in assembled components is presented. This method is based on the fusion of regions and edges information, and it offers a certain degree of simplification for the recognition and modelled of the disassembly tasks, of the set of components which compose the product. The proposed approach to detect regions is a hybrid approach between RGB and HSV spaces. The bi-dimensional histogram V/S is employed for the selection of the appropriate thresholds which serve as an aid to diminish the influence of the highlights and shadows in images. The goal of this paper is to present an approach for the detection of occlusions in assembled components from a combination of HSV and RGB spaces, a bi-dimensional histogram and an edge detector.
\end{abstract}

Keywords Assembled components · Bi-dimensional histogram $\cdot$ Colour segmentation $\cdot$ Colour space $\cdot$ Edges $\cdot$ Overlapping $\cdot$ Thresholding $\cdot$ Partial occlusions $\cdot$ Regions

P. Gil $(\bowtie) \cdot$ F. Torres · F. G. Ortiz

Department of Physics,

Systems Engineering and Signal Theory,

University of Alicante,

Crta. San Vicente $\mathrm{s} / \mathrm{n}$.,

P.O. Box 99 Alicante, Spain

e-mail: pgil@dfists.ua.es

Tel.: +34-965-903682

Fax: +34-965-903682

O. Reinoso

Department of Industrial Systems Engineering,

Miguel Hernandez University,

Alicante, Spain

\section{Introduction}

In industrial applications, computer vision techniques have been, and are being widely used. They also play an important role in many fields as well as in disassembly tasks. In robotic settings of disassembly systems, two important problems arise regarding computer vision: recognition and location $[1,2]$.

The designing and building of systems or machines which are able to perceive and interpret a complex scene as a set of assembled components which compose a product is a challenge for industry and man. Thus, man tries to build systems for interpreting, in a similar way to a human eye and brain, the images captured by cameras. It has been and will be an important field of research because objects recognition is an extremely complicated task.

The typical problems in objects recognition have been widely treated in Literature [3-5], and a lot of them take a special importance when the recognition process imply to identify a set of assembled components of an object [6-8]. In these cases, difficulties such as occlusions, deformations, highlights or shadows, appear.

The recognition of assembled object-components from images of cameras is limited when the system is not able to see all their area. Thus, an object-component can cover a portion of area of another object-component which has to be identified to disassemble it, with the disadvantage this one is not absolutely visible. This problem is known in computer vision like overlapping. Moreover, a component can hide part of itself by its own geometry. This is the property of opacity of a lot of objects.

It is necessary not to forget that an object or component can partially be occluded by its shadow or the shadow of another object which makes difficult the interaction with the first one. Furthermore, problems such as the produced highlights when the light affects certain surfaces make difficult to determine the type of component due to the loss of the homogeneity feature of the object surfaces.

In particular, the occlusions acquire certain importance when a system of vision, on the one hand, looks for determining if they exist or not in the object-component 
that compose an assembly. And, on the other hand, once the occlusions have been detected by the system, determining what part of the object-component has to be required to extract the features of the image, and compare with some models of object-components to resolve the recognition process.

This paper is organized as follows. Section 2 introduces the colour spaces and bi-dimensional histogram used to segment the colour images. In Section 3, we discuss the thresholding of the images and the differences between the segmentations depending on the colour space employed, RGB, HSV or hybrid approach RGBHSV. Section 4 describes the way to detect zones of possible occlusions or overlappings from the data obtained by the segmentation and the edges detection. Finally we summarize our work and propose some future work in Section 5.

\section{Colour spaces and segmentation tools}

A colour space is a three-dimensional body used to represent some colour organization according to a particular choice of three coordinates that describe colour. The representation of the colour is not a trivial problem, and its election influences the way the colour is used and its effectiveness for segmentation process. From this fact, colour has been organized in different colour spaces. In this section, we introduce two different colour spaces, RGB and HSV $[9,10]$, employed for segmenting several objectcomponents from captured images in a disassembly process. The colour segmentation has been widely studied, and a lot of research about this field appears in the literature [11-13]. Such spaces are commented on next.

\subsection{Representation of colour}

RGB space is the colour space better known and used in most video and photography colour cameras. Working with the same colour space that video cameras employ to capture and digitalize images is very interesting, because it permits to avoid the modification of the features of the colours during the information retrieval in segment processes. The typical mistakes, source of these modifications, are caused by the conversion and transformation processes. In addition, working with this space has the advantage of reducing the processing speed due to the saving of operations in the computation.

RGB space is represented geometrically as a cube where each colour is defined by the mixture of intensity values of the three primary colours, red, green and blue. Each colour is determined by an array of 3-coordinates. The black is represented with $(\mathrm{r}, \mathrm{g}, \mathrm{b})=(0,0,0)$ and the white is $(\mathrm{r}, \mathrm{g}, \mathrm{b})=$ $(255,255,255)$. And, the achromatic range of the grey scale is identified on the cube diagonal.

The HSV model is another approach of colour space [10], but it has a more classical and intuitive representation among all shown in the literature in the last years. Its geometric interpretation is represented by a cone with quasi-hexagonal base. With this colour space, each colour is defined by three basic components: hue, saturation and intensity of brightness. The hue, $h_{H S V}$, determines the value of chromaticity or type of colour, the saturation, $\mathrm{s}_{H S}$, references the wavelengths, which added to colour frequency, determine the amount of white into a colour. The less saturated the whiter this colour will be and the more saturated the less white it will be. Then, the saturation represents the purity and intensity of any colour. Thus, the lack of saturation is given by the axis of the HSV cone and it represents the grey scale from white to black. The intensity of brightness, $v_{H S}$, identifies the subjective appreciation of clarity or darkness. The HSV model is one of so many models of type LHS (lightness, hue, saturation) based on the mix and it specifies colours using tints, shades, and tones.

When a colour image has to be represented with the HSV model it is necessary to determine how the colour components of any RGB video camera influence in relation to its HSV space. Since, the HSV model is defined from RGB model, by:

$$
\begin{aligned}
& h_{H S V}= \begin{cases}\frac{g-b}{\max (r, g, b)-\min (r, g, b)} & \text { if } r=\max (r, g, b) \\
\frac{b-r}{\max (r, g, b)-\min (r, g, b)}+2 & \text { if } g=\max (r, g, b) \\
\frac{r-g}{\max (r, g, b)-\min (r, g, b)}+4 & \text { if } b=\max (r, g, b)\end{cases} \\
& s_{H S V}=\frac{\max (r, g, b)-\min (r, g, b)}{\max (r, g, b)} \\
& v_{H S V}=\max (r, g, b)
\end{aligned}
$$

Furthermore, we use the HSV colour space because it represents the saturation better than other models LHS such as HLS, although it has worse representation of the intensity of brightness. HLS is geometrically a double cone whose vertices identify the maximum and minimum values of brightness.

\subsection{Bi-dimensional histogram, V/S}

The histogram is probably one of the most widely tools used to analyze the information of each component of colour (or grey scale) from images. The classic histogram shows the concentration of the grey levels in a digital image. A histogram of a colour image is a set of three histograms where each colour component has its own representation which shows the distribution of its pixels in relation to the value of colour intensity. Unfortunately, the classic histogram does not relate information among components of colour. However, the bi-dimensional histograms are able of obtaining a relation between two given components. This type of histogram acquires certain importance when segmentation methods based on HSV space are applied. 
In particular, it is important to point out two bi-dimensional histograms commonly called as $\mathrm{V} / \mathrm{S}$ and $\mathrm{H} / \mathrm{S}$ histograms. In this paper, we have only used the V/S histogram.

Since, the V/S bi-dimensional histogram is a histogram with two Cartesian variables: value of brightness intensity and saturation. Its aim is to provide a simple and intuitive tool for performing partitions of chromatic information separating it from the achromatic $[14,15]$. The analysis of this histogram and the application of thresholds allow us to extract highlights and different levels of shadows. Therefore, the thresholding with small saturation threshold levels detects regions of the image with dull colours, for example the shadows and the brightness with low intensity value. A highlight could be detected choosing a low saturation value and a high intensity value, and a shadow could be segmented taking a low saturation value and a low intensity value. The building and analysis of V/S bi-dimensional histogram help to verify the distribution of highlights and shadows in images (Fig. 1).

\section{Segmentation multi-threshold combined}

\subsection{Segmentation with RGB and HSV spaces}

The segmentation used with RGB or HSV spaces is based on the detection of thresholds from the map of colour components RGB or HSV respectively. In robotic environments, the segmentation process by thresholding is often complex due to the lack of a previous knowledge. Thus, problems such as the difficulty to know the number of components which compose the object or objects, the effect of undesired shadows and highlights, the fact that the colours can be more or less complex, and the several kinds of textures and size of objects as well as the overlapping between object-components among other many situations can complicate this process.

This means that the election of a small number of thresholds causes the sub-segmentation, and consequently less wished regions are detected, or the election of many thresholds forces to the over-segmentation, which originates more regions of those that can be of our interest. Both situations can take place due to two reasons: the existence of shadows and highlights, and the similarity of the colour objects or of the object-components which compose them.
The thresholding of each colour component, RGB or HSV, has been carried out by means of the automatic detection of several thresholds with the Otu's method [16]. The Otsu's method is based on statistical and spatial information and it requires that the histogram consists of only Gaussian distributions. This method has to select a set of thresholds. The thresholds are used to divide the pixels of an image into several region-classes. We have considered one, the minimum number of thresholds for each component, and three the maximum. It has been done in this way because, at least, it is possible to separate an object from the background in all colour components and this can be made with only one threshold. And the maximum number is three because there are 64 possible region-classes when 3 thresholds for each colour component are used. The 64 region-classes are sufficient to segment an image of an assembly. The number of different region-classes is determined by:

$n C=\prod_{k=1}^{n c}\left(n u_{k}+1\right)$

where $n c$ indicates the layer number in image. For colour images, there are always 3 layers and they correspond to the basic components: red, green and blue in RGB, or hue, saturation and brightness intensity in HSV. And, $n u_{k}$, references the number of thresholds detected for each component.

The methodology used is derived from statistical and spatial information of histograms. The selection is a variation of the conventional Otsu's method [16], and it is based on the concepts of within-class, between-class variances and Gaussian's distributions. The computation of thresholds levels is calculated with a recursive approach to automate Otsu's method [17]. The recursive method allows us to segment without any constraints on the number of object-components as it happened in the method presented in [13].

The multilevel thresholding used permits to discriminate image pixels by dividing the original image into several region-classes, $C_{O}=\left\{1, \ldots, u_{1}\right\}, C\left\{u_{1}+1, \ldots, u_{2}\right\}, \ldots$, $C_{n}=\left\{u_{n}, \ldots, L-1\right\}$, where $L$ is the maximum value of intensity in the image, and where each region-class groups all the pixels with similar value of intensity to reach threshold detected by Otsu's method. The set of thresholds
Fig. 1 The V/S Histogram for HSV-colour space and its relations with shadows and hightlights
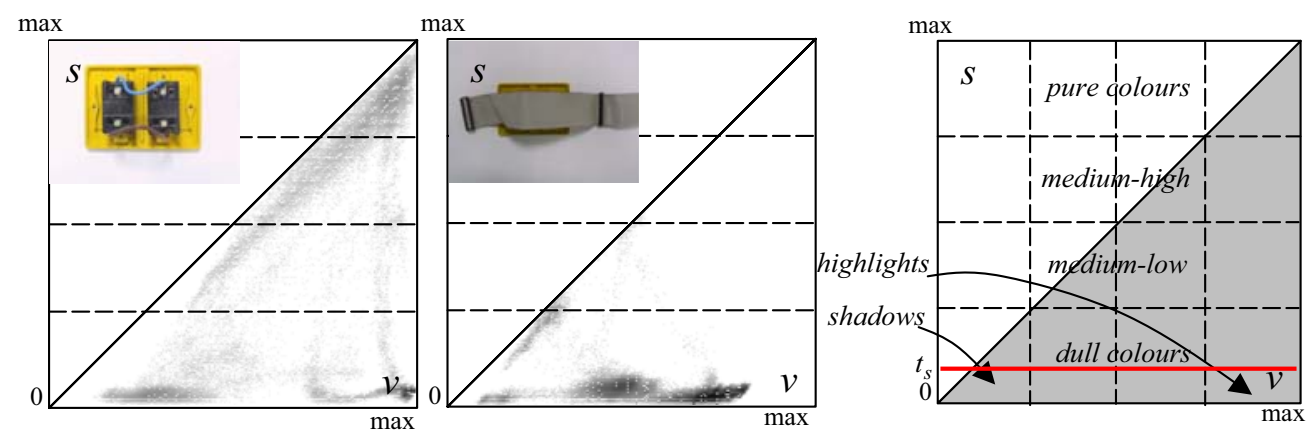
detected is denoted by $U=\left\{u_{1}, u_{2}, \ldots, u_{n}\right\}$. The way Otsu chooses the optimal thresholds, $U$, in order to partition the region-classes, so that the variance between region-classes, $\sigma^{2}$, is maximized, $U=\underset{1 \leq u_{1}<u_{2}<\ldots<u_{n}<L}{\arg \max }\left\{\sigma^{2}\right\}$; that is:

$\sigma^{2}=\sum_{k=1}^{n} \omega_{k}\left(\mu_{k}-\mu_{T}\right)^{2}$

where,

$\mu_{k}=\sum_{i \in C_{k}} i \cdot p_{i} / \omega_{k}$

$\omega_{k}=\sum_{i \in C_{k}} p_{i}, \omega_{k}=\sum_{i=1}^{u_{1}} p_{i}, \ldots, \omega_{n}=\sum_{i=u_{n+1}}^{L-1} p_{i}$

$\mu_{T}=\sum_{i \in C_{k}} \mu_{k}=\sum_{i=1}^{L-1} i \cdot p_{i}$

Let, $\mu_{K}$, be the mean intensity for classes, $C_{K} ; \omega_{K}$, the probability that a pixel belongs to the class, $C_{K}, \mu_{T}$, the mean intensity for the whole image, and $p_{i}$ are pixels with value of intensity $i$.

The segmentation from RGB image is based on thresholding each colour component using the set $U$, separately, but previously each colour component has been smoothed to improve each histogram and thus obtaining the thresholds with more success and precision. The smoothing is realized by averaging of the values of intensity from image pixels contained in a $5 \times 5$ neighbourhood.

Therefore, three sets of thresholds will be obtained. So, the number of region-classes, in which the image has been partitioned, is determined as stated in (2). In the segmentation process, the colours are assigned according to the values of intensity, $\widetilde{p}_{i j, k}$, for each colour component, $k$, depending on the number of thresholds obtained, $n u_{k}$. Figure 2 shows the thresholded and coloured process from sample image for each colour component.

$\widetilde{p}_{i j, k}=\left\{\begin{array}{clc}0 & \text { if } & p_{i j, k} \leq u_{1, k} \\ \frac{L}{n u_{k}} \cdot n & \text { if } & u_{n, k}<p_{i j, k} \leq u_{n+1, k}, n=1 \ldots n u_{k} \\ L-1 & \text { if } & p_{i j, k}>u_{n u_{k}, k}\end{array}\right.$

Afterwards, we joint each thresholded colour component with Otsu's method and we colours them according to Eq. (7). In addition, each pixel of segmented colour image is codified as $C=\left\{c_{1}, c_{2}, c_{3}\right\}$, where $c_{k}$ can take only values from $\widetilde{p}_{i j, k}$, and, the different codifications determine a possible region-class, $\left\{C_{k}\right\}_{k=1}^{n C}$. Figure $2 \mathrm{~b}$ shows the resulting image after thresholding of each colour component, and Fig. $2 \mathrm{~d}$ shows the clustered image after the images represented in Fig. $2 b$ have been jointed and coloured in a segmented image (Fig. 2c).
Once the original image has been segmented (Fig. 2c), it is necessary to reduce the number of region-classes, $n C$, since an excessive segmentation can partition an image up to 64 region-classes. Then, we fuse region-classes using a cluster process based on statistical concepts and criteria such as variance, average and Euclidean distance (Fig. 2d). This process is similar to the one commented in [13] but generalized for an undetermined number of thresholds, $n c$.

Another approach employed to segment images is based on applying the same principles used for RGB but using the HSV model. The first step is the transformation from the RGB image to HSV image, obtaining its three colour components (hue, saturation and brightness intensity). These three resultant monochrome images are analyzed to make the segmentation.

The detection of objects and their components from colour images captured in scenes where a robot manipulates them, for example in disassembly tasks, is too complex. For this reason, and to simplify the detection problem, we have supposed that the lack of chromaticity from colour images acquired is due to two basic situations: existence of zones of shadows and zones of highlights in the image. This type of information (shadows and highlights) is analyzed with the construction of the V/S bi-dimensional histogram. The V/S histogram determines according to its distribution of saturations and brightness values, what thresholds are the most suitable not to consider shadows and highlights. If the set of Otsu's thresholds detected is $U=$ $\left\{u_{1}, u_{2}, \ldots, u_{n}\right\}$ and the distributions of saturations and brightness determine $t_{s}$. The factor, $t_{s}$, is the threshold which delimits the zones with low saturation, where the dull colours appear (Fig. 1).

On the one hand, it is very important to emphasize that, if a component or object has a zone of shadows, it must not be added as if it was part of the area of the component or object. On the other hand, a same component or object with different zones of shadows must not be segmented as different region-classes. The HSV space colour provides simpler and more intuitive information about lack of chromaticity than the RGB space. The low saturation defines this lack of chromaticity.

Considering a similar scheme to the one used in the RGB segmentation, the algorithm steps are shown as follows:

1. Transforming RGB to HSV image (Fig. 3a) to separate each component, $\mathrm{H}, \mathrm{S}, \mathrm{V}$, and smooth them to eliminate discontinuities into the histogram. Eq. (1) is used to this transformation.

2. Detecting the Otsu thresholds, $n u_{k}$, for each component $k=\{H, S, V\}$ (Fig. 3b). We have used Eqs. (3) to (6), and have maximized $\sigma^{2}$.

3. Selecting the most suitable thresholds for the chromatic segmentation among all the detected ones by Otsu's method. This is done by means of V/S bi-dimensional histogram and fixing the zone delimited by $t_{s}$ (Fig. 1).

4. Each pixel is codified with a code $C=\left\{c_{1}, c_{2}, c_{3}\right\}$, where $c_{k}$ takes value using the Eq. (3). Thus a $C_{K}$ set of region-classes is formed (Fig. $3 \mathrm{c}$ ). 
Fig. 2 a Original image of assembled object-components. b Thresholding for each component of colour. c Detected regions by segmentation RGB. d Clustered Regions

Fig. 3 a Original image of assembled object-components. b Thresholding for hue, saturation and intensity. c Detected regions by HSV segmentation. d Clustered Regions by means of hue

Fig. 4 a Original image of assembled object-components. b Detected regions by RGB-HSV segmentation and clustering
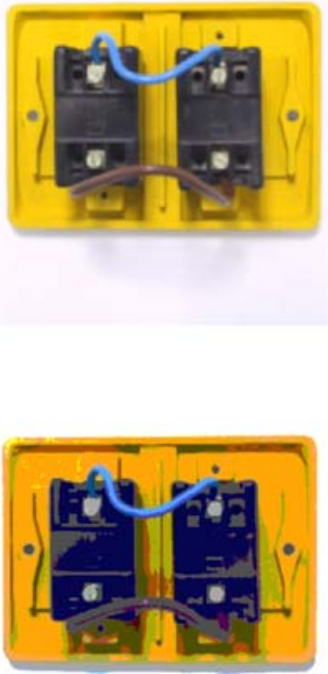

a
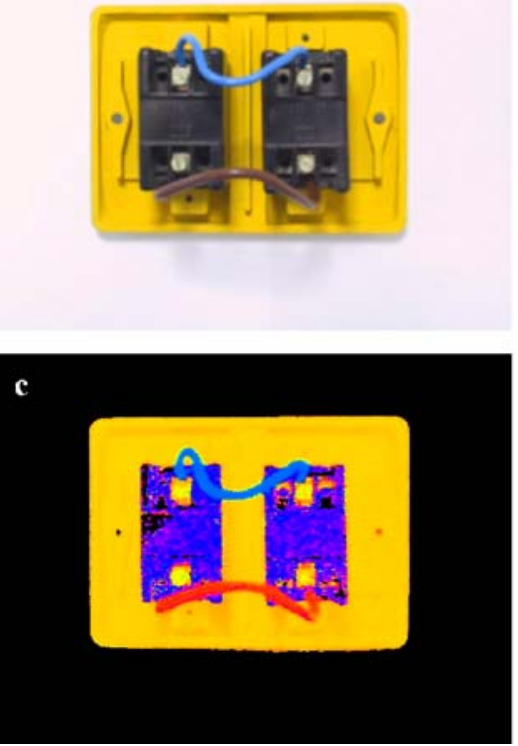
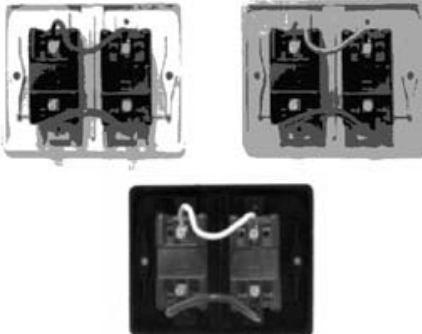

d

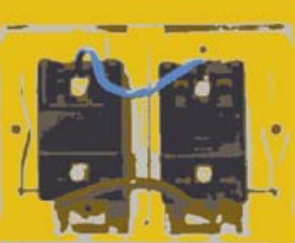

b
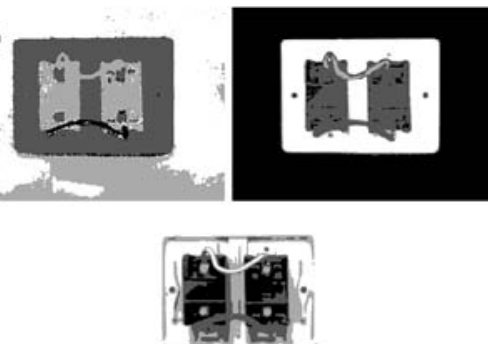

\section{d}

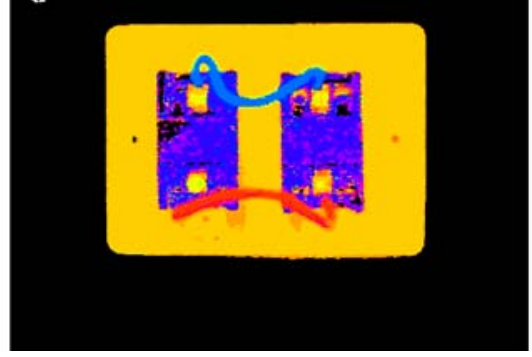

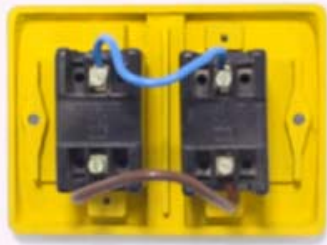

b

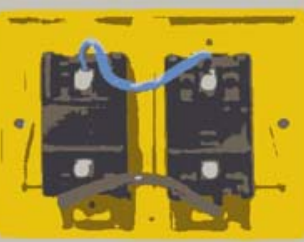


5. A cluster process is applied to reduce the number of region-classes. This is done looking for homogeneity regions and grouping them. The criterion to fuse the grouped regions is simpler than in the RGB model. Only, the variance measures and standard deviation with respect to average value of chromaticity are used. The information of chromaticity is kept into the polar component of hue, $\mathrm{H}$. This step is applied for each region-class detected at step 4.

6. The clustered region-classes are coloured, allocating as colour the average of value of hue for each one (Fig. 3d).

An analysis and interpretation of segmented image with the three different approaches, shows the impossibility to avoid a bad segmentation due to shadows and highlights (Fig. 2d) with RGB segmentation. This is because the three colour component $(r, g, b)$ represent intensity values. In contrast, the second method based on HSV and the V/S histogram solves this problem but it requieres that the colours are transformed for display (Fig. 3d) and there is a lost of realism because it is only considered the information of hue for clustering.

However, the hybrid RGB-HSV segmentation combines these two colour spaces to segment and it overcomes the disadvantages of both. (Fig. 4b). Thus, the hybrid approach can hadle information to segment images with shadows and highlihts thanks to V/S histogram and in addition it can cluster without an excessive loss of real colour.

\subsection{Segmentation with RGB-HSV joined}

The advantage that offers the RGB segmentation multithreshold is based mainly on its capacity to detect chromatic information. This information can have diverse tones of colour including the achromatic, grey scale. For example, it is possible to detect object-components whose colour is a tone of grey: these are the screws in Fig. 2, but also, the RGB segmentation forces to detect regions of shadows and highlights in same way that these regions were objectcomponents. In this type of segmentation, the shadows and highlights are considered object-components of a certain grey colour when they are not that (Fig. 3c,d).

Nevertheless the HSV method shown previously, permits to detect colour objects without considering the zones of shadows and highlights in images. These zones are rejected as object-components valued due to its achromatic characteristic. Sometimes, this supposes a disadvantage since an object or component of grey colour will be not detected correctly because it will be considered as either shadow or reflect. This case is shown in Fig. $3 a-c$ where it is possible to observe that the screws have not been detected. In addition, the HSV method works with its three components (hue, saturation and brightness) disconnected. This fact causes a loss of the general view of colour information. The three components of HSV space are not associated among them and a major difficulty of segmentation and cluster processes of colour image have been shown. Fig. $3 d$ shows a segmented image with colours very different to its sample image shown in Fig. 3a.

A combination of the RGB and HSV methods has been chosen after proving both methods separately. This new method consists of two steps. Initially, the objects or theirs components in the image are detected using the RGB segmentation commented in the section 3.1. And finally, a post-segmentation from RGB segmented image is realized using the information that provides the components of the HSV space and the analysis of the V/S histogram (Fig. 1).

Thus, the new segmentation searches for detecting all the objects in the image although these almost do not have chromaticity, as well as it has also the aim of eliminating the shadows and highlights as far as possible to obtain regions more homogeneous.

As can be seen in Fig. 4, the result of combining both colour spaces, guarantees more realistic and not so artificial colours as those obtained when the HSV model or RGB model is only used. In the RGB-HSV model, the clustering process is carried out with the three colour components of the RGB space. This way, the colours are less synthetic and more similar to the colours of the original image. Furthermore, the use of components of HSV space and the V/S bi-dimensional histogram corrects some situations of illumination, highlights on the background and slight shadows, permitting more successful segmentations. The new combined method improves the clustering process, avoiding the fusion of not homogenous regions which can appear in the single RGB segmentation, as can be seen in Figs. 4b and 5 .

Figure 5 shows the experimental results of applying the method HSV-RGB colour segmentation from sample image represented in Fig. 4a. The number of detected region-classes has been 16 of 64 with 3 thresholds for each component. And the clustering process has reduced to 6 region-classes.

\section{Detection of occlusions of assembled components}

Once the object components have been segmented and separated by regions of colour, it is important to identify the possible contact zones among the different objectcomponents which compose the assembly. These contact zones determine the candidate parts of regions where there can be an overlapping and/or occlusion. Thus, first, it is necessary to delimit the pre-search regions where there can be contact among object-components in the segmented images. And, second, to eliminate the pre-search regions which do not correspond to overlapping zones among object-components, according to its characteristics. This way, the pre-search space is restricted for reducing the amount of false zones of overlapping or occlusion.

Therefore, in this stage of processing, an edge detector is used to restrinct and delimit the pre-search regions to the zones where there are significant changes of colour. In order to locate the pre-search regions where there are possible overlappings, a space of search formed by all those zones where the gradient of the colour components is a 
Fig. 5 RGB-HSV segmentation and clustering process for the assembly of Fig. 4
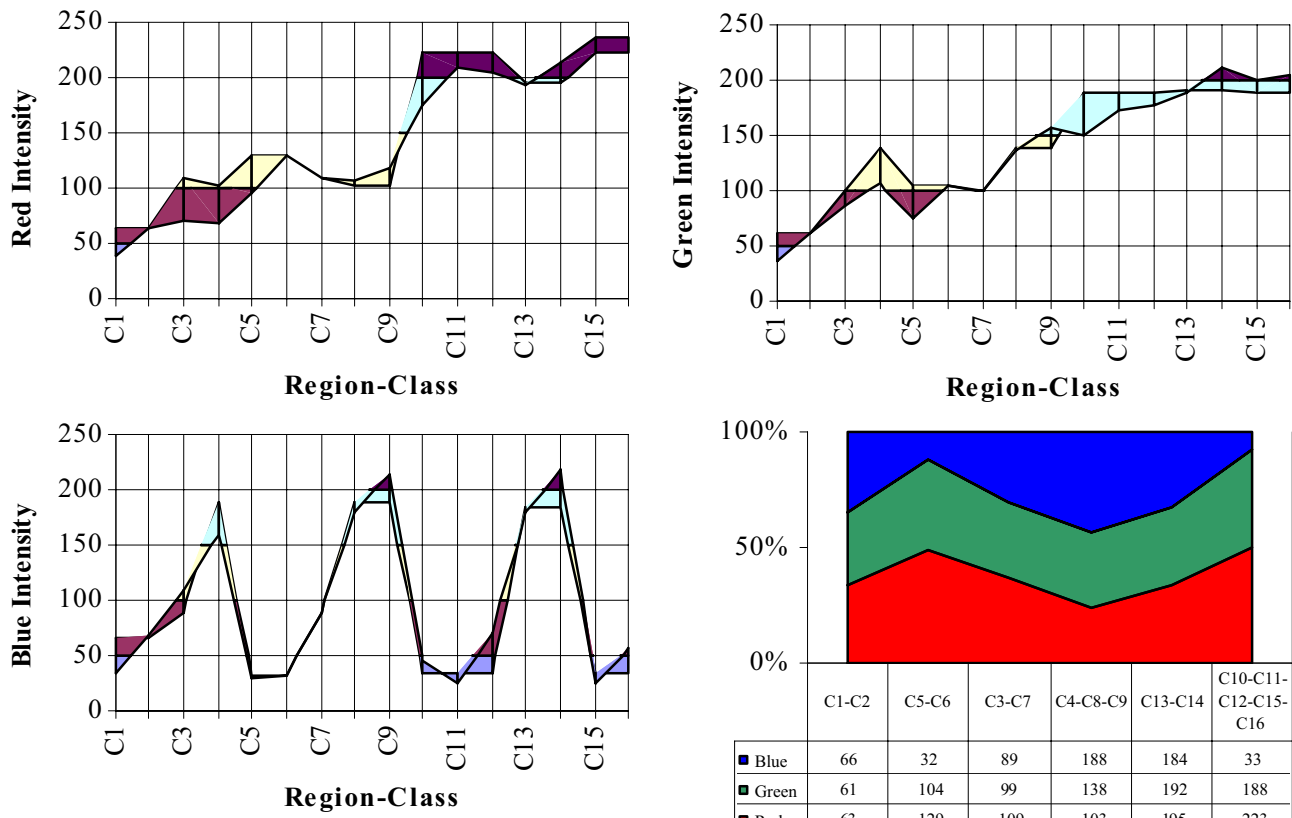

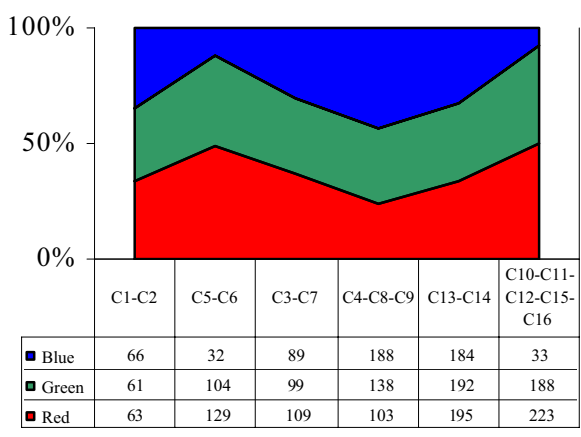

local maximum, is computed. Then, the pre-search regions are determined by the pixels pertaining to detected edges using the Canny's detector [18]. In the literature, there are other edge detection methods as the Cumani's detector [19]. The principal advantage of Cumani's detector is the detection of edges in multispectral images, such as RGB images. We have considered the Canny's method instead of Cumani's method for computational cost reasons. An optimal detection by the Cumani's detector needs the three colour components and Canny's detector only works with the componet of intensity and therefore this last one is more express to execute. The intensity is $i=(r+g+b) / 3$ and $\nu=\max \{r, g, b\}$ for RGB images and HSV images, respectively.

Nevertheless, the Canny's detector considers edges which do not belong to overlapping and/or oclusion. For this reason, once calculated the edges of Canny which determine the pre-search space, the edges whose characteristics determine that are not of interest are rejected. The rejected edges are those which can never belong to overlapping zones and/or occlusion.

Thus, an edge is rejected and not considered like part of an overlapping when it separates any colour region of another region that has the colour of the background. The colour regions and colour of the background are computed according to Section 3. That is to say, the rejected edges are those which are part of the contour of all the set of objectcomponents that compose the assembly. To do it, the presearch space is reduced to the proximity of the edges which are inner to the object-components of the assembly. Since, these inner edges are the limits of candidate overlapping regions.

Concluding, there are two types of edges that belong to the pre-search space of overlapping. On the one hand, the edges which constitute the contour of the assembly like an object made up of a set of object-components, called external edges, and, on the other hand, the edges of the contour of each object-component, called internal edges. The occlusion is generally part of an overlapping and these phenomenona are determined by the detection of internal edges. Therefore the external edges can be rejected to compute the occlusion zones.

Figure 6, shows an example of detection for occlusion zones among components of an assembly.

Nextly, the steps to explain the process of detection and identification of overlappings with occlusion, are detailed:

1. To read segmented image in Section 3: This image represents each object-component that compose the assembly of an object separated among themselves and separated of background.

2. To detect internal and external edges using the Canny's detector.

3. To link the calculated edge points by Canny's detector. The edge points are joined into lists of sequential edge points, during a labelling process. Thus, there is one list for each edge of contour discarding the contours with a small amount of pixels.

The process consists in scanning through the image of Canny searching of edge points. When, an edge point is found, the rest of near edge points are looked for using a 8-connected mask. Then, all found edge points, around the neighbourhoods in the same direction of search are labelled like points of the same contour. The objective is checking all the edge points associated with a start point edge and grouping them if they are in the same direction of search, until there are not any points in that direction. When no more points can be connected throughout that direction of search, the process returns to the start point and tracks in the 
Fig. 6 Detail of overlapping with occluded partially region
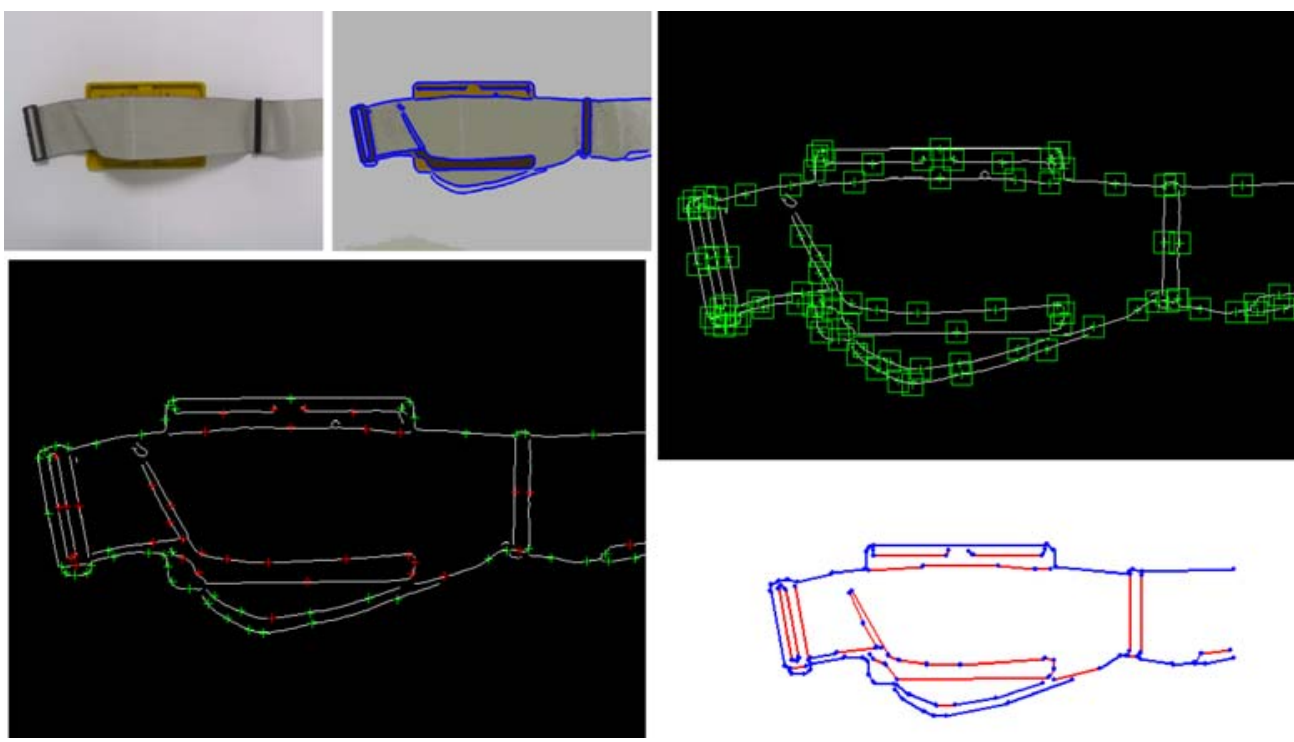

opposite direction. Finally, a check for the overall number of edge points found is made and the edge is ignored if it is too short.

4. To fit the contours (labelled linked edge points) by means of straight line segments. The fitting is made using the distance between pixels as a criterion.

5. To merge segments. In this step, the straight line segments are checked seeking to merge segments together. The segments are merged if the orientation difference is less than orientation angle of reference, and the ends of the segments are a distance smaller than a given tolerance.

6. To distinguish internal edges and external edges. In order to be able to differentiate them, the segments of contour are tracked and its colour values of its neighbourhoods are tested for each one of its edge points. With the purpose of reducing the computational cost, three significant points are only used throughout each segment of contour, the ends and the midpoint between them. For each point, the number of different colours within a vecinity area is verified. The number of these colours and its type of colour determine if the edge is internal or external. Whenever an edge is external it is rejected like overlapping zone. And, if an edge is internal, then it is verified if it determines a possible overlapping zone. An edge point belongs to an external edge if there is a significant percentage of pixels with the same colour as the background. It is important to emphasize that during the segmentation process, failures can appear due to little homogeneity in the colour of the segmented object-components. This problem is avoided by not considering the colours with percentage of pixels below a quota of tolerance (aprox. $0.15 \%)$.
Fig. 7 a Edges detector. b Edge points for searching. c Types of detected edges points. d Types of edges with overlapping and possible occlusions
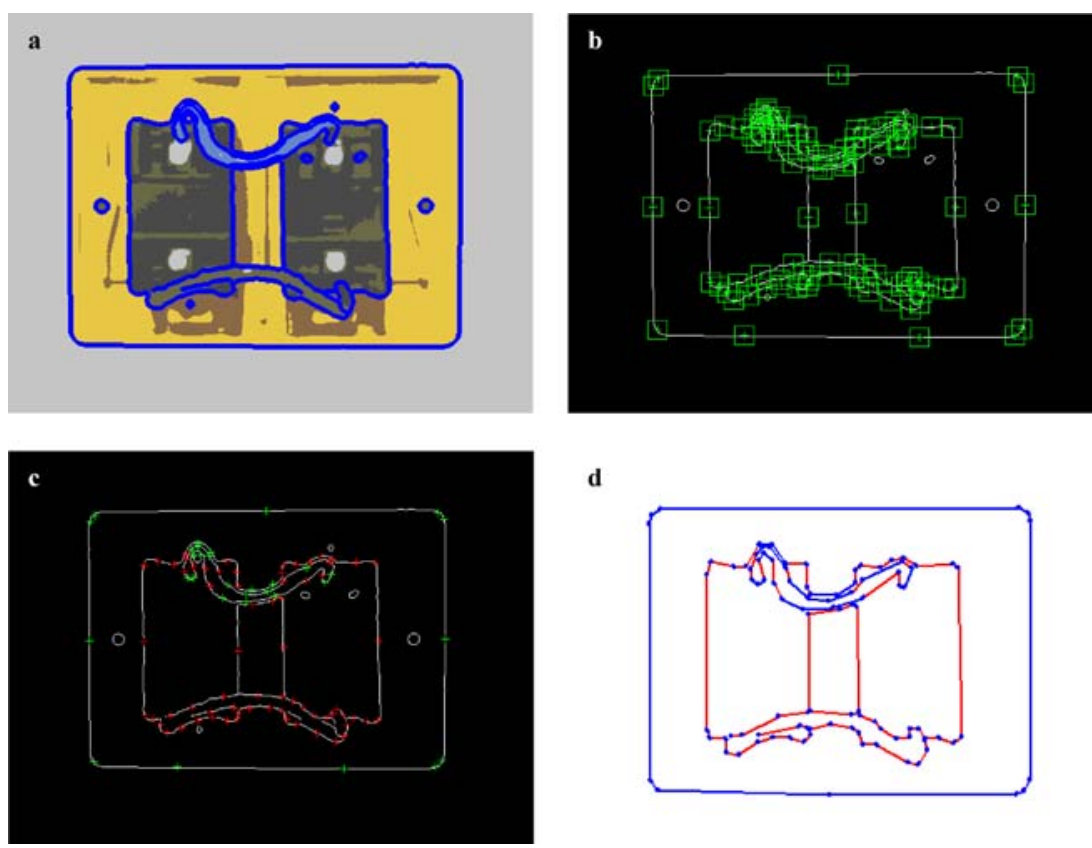
Fig. 8 Several views of the segmented assembly
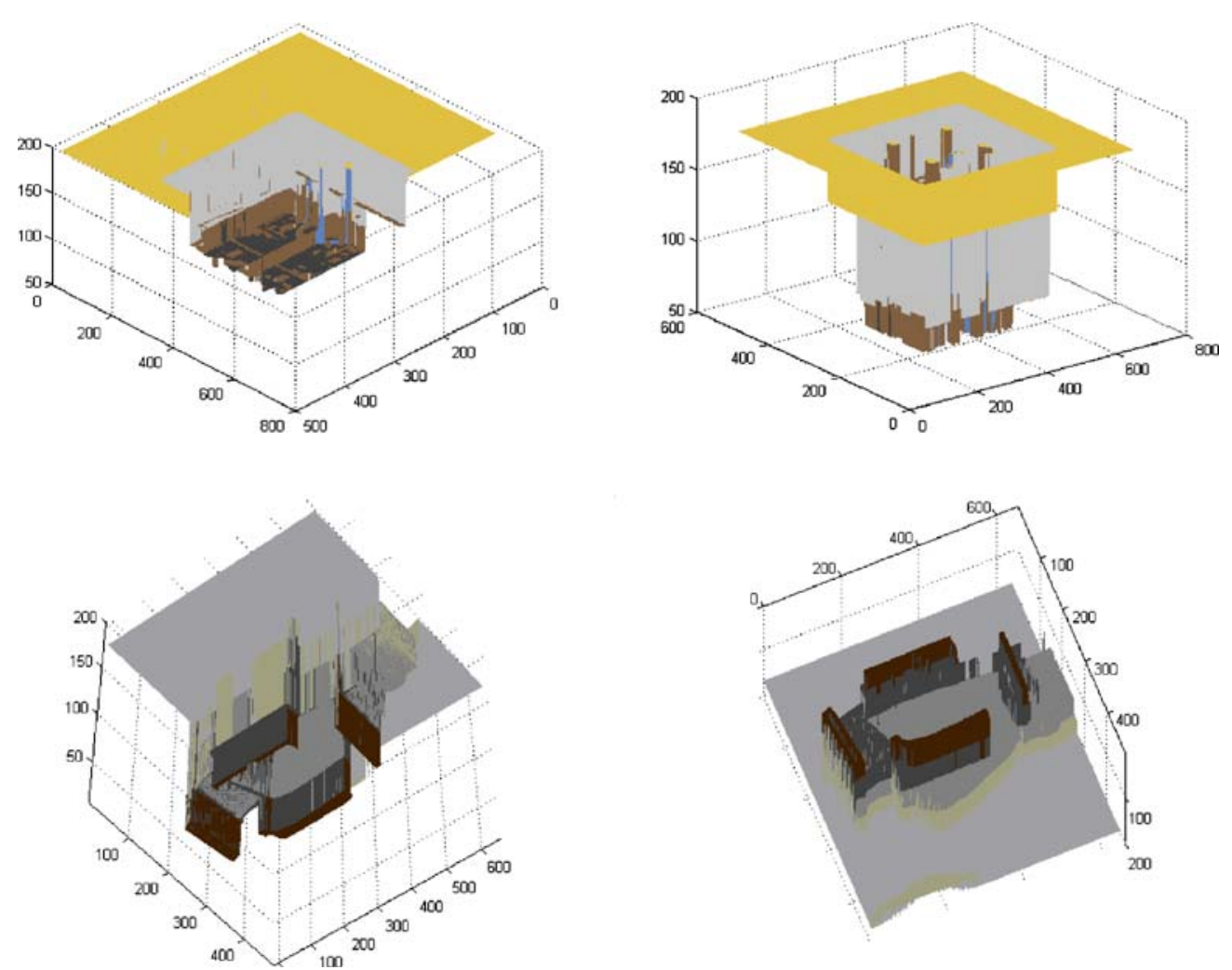

In Fig. 7, an example of detected partial occlusions is shown. This sequence of processing is determined by the above-described steps. Firstly, the edge points are detected and linked, afterwards, the space for searching is computed and, finally, the kind of edges with overlapping or occlusions are computed.

This method is constrained to use colour information, therefore the principal problem can be defined in terms of detecting the possible overlaping or occlusions in grayscale images. The principal motivations to work with colour images instead of using grayscale images is due to the difficulty to segment the highlights and shadows appearing in them. A shadow in grayscale can be confused with another level of darker gray or sure gray, respectively. For future projects, a possible solution to surpass this limitation consists in incorporating structured light to identify these types of regions. Furthermore, the use of the structured light will suppose a new motivation to improve the detection of occlusions in colour images. Structured light could help to distinguish between an occlusion without contact, in which an object-component hides another one depending on the viewpoint, and the oclussion with contact, in which two or more object-components overlap each other.

\section{Conclusions}

The artificial vision systems are an important part of the sensorization and supervision processes for disassembly tasks. Using computer vision techniques not only permits to determine which element is the product and which are theirs components to be disassembled, but also permits to improve the design and construction of the graphs [20] or schemes which model the sequence [21, 22] of tasks during the disassembly. In this work, an approach based on region segmentation combining the RGB-HSV colour spaces and an edges detector is presented to detect overlapping and occlusion zones in disassembly tasks.

The presented method implements a multi-thresholding segmentation in each of the components of a RGB-colour image and it uses the information of hue, saturation and intensity of its equivalent HSV-colour image which have been segmented with multi-thresholding, too. This method is novel because it combines information about the two colour spaces HSV and RGB. In addition, it uses tools as the bi-dimensional histograms V/S to segment better with certain chromaticity.

Furthermore, the experiments demonstrate that the method RGB-HSV colour segmentation obtains better results than the single HSV or RGB segmentation more often. That is to say, for RGB-HSV segmentation, the colours obtained are as realistic as in the simple RGB, and they do not have so many problems of shadows and colours fusion in the clustering stage that union significant region-classes produce (Figs. 4 and 8). This fact facilitates the recognition and supervision of the objects and its components during the disassembly tasks.

Finally, an approach mixing the detected edges and segmented regions with the RGB-HSV is presented to detect overlapping and partial occlusions. The detection of overlapping and/or partial occlusions simplifies the disassembly process because it reduces the complexity of the graph that models the movements' sequence of disassem- 
bly and, in addition it improves the recognition process. This is the reason of that the disassembly order for each object-component depends on how many object-components are located upon others.

Acknowledgements This work was funded by the following Spanish MCYT project "DESAURO: Desensamblado Automático Selectivo para Reciclado mediante Robots Cooperativos y Sistema Multisensorial” (MCYT (DPI2002-02103).

\section{References}

1. Torres F, Gil P, Puente ST, Pomares J, Aracil R (2004) Automatic Pc disassembly for component recovery. Int J Adv Manuf Technol 23:39-46

2. Gil P, Puente ST, Torres F, Pomares J, Candelas FA (2001) Data fusion from multiple cameras for automatic disassembly, Proceedings, IFAC Workshop of Intelligent Assembly and Disassembly (IAD'01), Canela, Brazil 85-90

3. Koch MW, Kashyap RL (1987) Using polygons to recognize and locate partially occluded objects. IEEE Trans Pattern Anal Mach Intell 4(9):483-494

4. Ullman JR (1993) Edge Replacements in the recognition of occluded objects. Pattern Recog 12(26):1771-1784

5. Ulrich M, Steger C, Baumgartner A, Ebner H (2001) Real Time Object Recognition in digital images for industrial applications. $5^{\text {th }}$-Conference on Optical 3D measurement techniques, Wien, Germany 308-318

6. Najjari H, Steiner SJ (1996) 3D CAD-based object recognition for a flexible assembly cell, in Intelligent Robots and Computer Vision XV: Algorithms, Techniques, Active Vision, and Materials Handling 2904:167-177

7. Xiong Y, Quek F (2002) Machine vision for 3D mechanical part recognition in intelligent manufacturing environments. IEEE 3th - International Workshop on Robot, Motion and Control (RoMoCo'02), Bukowy Dworek, Poland 441-446
8. Bauckhage C, Braun E, Sagerer G (2004) From image features to symbols and viceversa-using graphs to loop data -and model-driven processing in visual assembly recognition. Int $\mathrm{J}$ Pattern Recogn Artif Intell 3(18):497-517

9. Palus H (1998) Representations of colour images in different colour spaces. Sangwine S et al (eds). The Colour Image Processing Handbook. Chapman and Hall pp 67-90

10. Smith AR (1978) Color gamut transformation pairs. Comput Graph 3(12):12-19

11. Pal NR, Pal S (1993) A review on image segmentation techniques. Pattern Recogn 26:1277-1294

12. Deng Y, Manjunath (2001) Unsupervised segmentation of color texture regions in images and video. IEEE Trans Pattern Anal Mach Intell 8(23):800-810

13. Yingzi D, Chein IC., Thouin PD (2004) Unsupervised approach to color video thresholding. Opt Eng 2(43) 282-289

14. Angulo J (2003) Morphologie mathématique at indexation d'images couleur. Application à la microscopie en biomedicine. Tesis doctor de la Ecole Des Mines de Paris

15. Torres F, Angulo J, Ortiz F (2003) Automatic detection of specular reflectance in colour images using the MS diagram. Lecture Notes in Computer Science 2756:132-136

16. Otsu N (1978) A threshold selection method from grey-level histograms. IEEE Trans Syst Man Cybern 8:62-66

17. Cheriet M, Said JN, Suen CY (1998) A recursive thresholding technique for image segmentation. IEEE Trans Image Process 6 (7):918-921

18. Canny JF (1986) A computational approach to edge detection. IEEE Trans Pattern Anal Mach Intell 6(8):112-131

19. Cumani A (1991) Edge detection in multispectral images. CVGIP Graph Models Image Process 53:40-51

20. Puente ST, Torres F, Pomares J (2001) Product disassembly scheduling using graph models. SPIE International Symposium on Intelligent Systems and Advanced Manufacturing, Newton, USA 4569:63-70

21. Torres F, Puente ST, Aracil R (2003) Int J Adv Manuf Technol 21(5):317-327

22. Pomares J, Puente ST, Torres F, Candelas FA, Gil P (2004) Virtual disassembly of products based on geometric models. Comput Ind 55:1-4 